\title{
Influence of cardiac autonomic neuropathy on cardiac repolarisation during incremental adrenaline infusion in type 1 diabetes
}

\author{
Alan Bernjak ${ }^{1,2}$. Elaine Chow ${ }^{3,4,5}$ Emma J. Robinson ${ }^{1,4} \cdot$ Jenny Freeman ${ }^{6}$ • Jefferson L. B. Marques ${ }^{1,7}$. \\ Ian A. Macdonald ${ }^{8}$. Paul J. Sheridan ${ }^{3,4} \cdot$ Simon R. Heller ${ }^{1,4}$ (D)
}

Received: 4 July 2019 / Accepted: 13 January 2020 / Published online: 7 February 2020

(C) The Author(s) 2020

\begin{abstract}
Aims/hypothesis We examined the effect of a standardised sympathetic stimulus, incremental adrenaline (epinephrine) infusion on cardiac repolarisation in individuals with type 1 diabetes with normal autonomic function, subclinical autonomic neuropathy and established autonomic neuropathy.

Methods Ten individuals with normal autonomic function and baroreceptor sensitivity tests (NAF), seven with subclinical autonomic neuropathy (SAN; normal standard autonomic function tests and abnormal baroreceptor sensitivity tests); and five with established cardiac autonomic neuropathy (CAN; abnormal standard autonomic function and baroreceptor tests) underwent an incremental adrenaline infusion. Saline $(0.9 \% \mathrm{NaCl})$ was infused for the first hour followed by $0.01 \mu \mathrm{g} \mathrm{kg} \mathrm{min}^{-1}$ and $0.03 \mu \mathrm{g} \mathrm{kg}^{-1} \mathrm{~min}^{-1}$ adrenaline for the second and third hours, respectively, and $0.06 \mu \mathrm{g} \mathrm{kg}^{-1} \mathrm{~min}^{-1}$ for the final $30 \mathrm{~min}$. High resolution ECG monitoring for $\mathrm{QT}_{\mathrm{c}}$ duration, ventricular repolarisation parameters $(\mathrm{T}$ wave amplitude, $\mathrm{T}$ wave area symmetry ratio) and blood sampling for potassium and catecholamines was performed every $30 \mathrm{~min}$.

Results Baseline heart rate was $68(95 \%$ CI 60, 76) bpm for the NAF group, $73(59,87)$ bpm for the SAN group and $84(78,91)$ bpm for the CAN group. During adrenaline infusion the heart rate increased differently across the groups $(p=0.01$ ). The maximum increase from baseline $(95 \% \mathrm{CI})$ in the CAN group was $22(13,32) \mathrm{bpm}$ compared with $11(7,15) \mathrm{bpm}$ in the NAF and $10(3,18) \mathrm{bpm}$ in the SAN groups. Baseline QT $\mathrm{C}_{\mathrm{c}}$ was $382(95 \%$ CI 374,390$) \mathrm{ms}$ in the NAF, 378 (363, 393) ms in the SAN and $392(367,417) \mathrm{ms}$ in the CAN groups $(p=0.31) . \mathrm{QT}_{\mathrm{c}}$ in all groups lengthened comparably with adrenaline infusion. The longest $\mathrm{QT}_{\mathrm{c}}$ was $444(422,463) \mathrm{ms}$ (NAF), $422(402,437) \mathrm{ms}$ (SAN) and $470(402,519) \mathrm{ms}(\mathrm{CAN})(p=0.09)$. T wave amplitude and $\mathrm{T}$ wave symmetry ratio decreased and the maximum decrease occurred earlier, at lower infused adrenaline concentrations in the CAN group compared with NAF and SAN groups. AUC for the symmetry ratio was different across the groups and was lowest in the CAN group $(p=0.04)$. Plasma adrenaline rose and potassium fell comparably in all groups.

Conclusions/interpretation Participants with CAN showed abnormal repolarisation in some measures at lower adrenaline concentrations. This may be due to denervation adrenergic hypersensitivity. Such individuals may be at greater risk of cardiac arrhythmias in response to physiological sympathoadrenal challenges such as stress or hypoglycaemia.
\end{abstract}

Alan Bernjak and Elaine Chow should be considered joint first authors.

Electronic supplementary material The online version of this article (https://doi.org/10.1007/s00125-020-05106-7) contains peer-reviewed but unedited supplementary material, which is available to authorised users.

Simon R. Heller

s.heller@sheffield.ac.uk

1 Department of Oncology \& Metabolism, University of Sheffield, Medical School, Beech Hill Road, Sheffield, UK

2 INSIGNEO Institute for in silico Medicine, University of Sheffield, Sheffield, UK

3 Department of Infection, Immunity and Cardiovascular Disease, University of Sheffield, Sheffield, UK

4 Sheffield Teaching Hospitals NHS Foundation Trust, Sheffield, UK
5 Present address: Department of Medicine and Therapeutics, the Chinese University of Hong Kong, Hong Kong, China

6 Leeds Institute of Life Sciences, University of Leeds, Leeds, UK

7 Present address: Institute of Biomedical Engineering, Department of Electrical and Electronic Engineering, Federal University of Santa Catarina, Florianópolis, SC, Brazil

8 School of Life Sciences, Queen's Medical Centre, University of Nottingham, Nottingham, UK 


\section{Research in context}

\section{What is already known about this subject?}

- Cardiac autonomic neuropathy (CAN) is a serious complication of diabetes, associated with high risk of cardiac arrhythmias and sudden death

- It could have a role in the 'dead-in-bed' syndrome through abnormal autonomic neural responses to nocturnal hypoglycaemia in young individuals with type 1 diabetes

- The counterregulatory sympathoadrenal response is a potentially important driver of abnormal cardiac repolarisation during hypoglycaemia

What is the key question?

- Does sympathetic stimulation in individuals with type 1 diabetes cause greater abnormal cardiac repolarisation in those with CAN compared with those without?

What are the new findings?

- Individuals with established CAN demonstrated greater increases in heart rate, despite comparable adrenaline and potassium concentrations

- Duration of cardiac repolarisation was longer in these individuals but this did not reach statistical significance, probably owing to the small numbers of people with CAN

- In those with CAN, abnormal changes in cardiac repolarisation were detected at lower adrenaline concentrations

How might this impact on clinical practice in the foreseeable future?

- These findings might in part explain why those with established autonomic neuropathy are at increased risk of sudden death

- Screening for autonomic dysfunction might identify patients with diabetes at increased risk of cardiac arrhythmia during hypoglycaemia

Keywords Adrenaline infusion · Cardiac autonomic neuropathy $\cdot$ Cardiac repolarisation · Type 1 diabetes

\section{Abbreviations}

BRS Baroreceptor sensitivity

CAN Cardiac autonomic neuropathy

NAF Normal autonomic function

SAN Subclinical autonomic neuropathy

\section{Introduction}

Cardiac autonomic neuropathy (CAN) is a serious complication of diabetes associated with increased rates of cardiac arrhythmias and sudden death [1]. Individuals with CAN exhibit intracardiac sympathetic imbalance due to cardiac parasympathetic denervation and initial sympathetic hypersensitivity leading to progressive sympathetic denervation [2]. These mechanisms might combine with the effects of hypoglycaemia to increase pro-arrhythmic risk and could identify CAN as a potential risk factor for sudden unexpected nocturnal deaths in type 1 diabetes, also known as the 'dead- in-bed' syndrome [3]. We previously tested this hypothesis in a study involving experimental hypoglycaemia in participants with type 1 diabetes [4]. Paradoxically, individuals with CAN showed smaller increase in QT interval duration compared to those with normal autonomic function. However, they also had attenuated sympathoadrenal responses.

In the current study, we aimed to investigate whether type 1 diabetic individuals with established autonomic neuropathy or subclinical autonomic neuropathy (SAN) would develop greater changes in cardiac repolarisation compared to those with normal autonomic function, when exposed to a standard sympathetic stimulus.

\section{Methods}

\section{Research design}

Individuals with type 1 diabetes, 14 men and eight women, aged between 18 and 50 years were recruited. Exclusion criteria included ischaemic heart disease, peripheral vascular 
disease, cerebrovascular disease, pregnancy, thyrotoxicosis, epilepsy or seizure disorder, asthma, visual impairment due to retinopathy, renal impairment due to nephropathy or current treatment with salbutamol or $\beta$-blockers. All participants gave written informed consent. The study protocol was approved by the North Sheffield Research Ethics committee.

Standard autonomic function tests were performed. See electronic supplementary material (ESM) Methods: Standard autonomic function tests for details. Responses outside ageadjusted normal ranges [5] in two or more tests were classified as abnormal standard autonomic function tests. Baroreceptor sensitivity (BRS) was calculated using the sequence method [6] (see ESM Methods: Baroreceptor sensitivity). BRS values lower than the 5th centile of age- and sex-adjusted ranges [7] were classed as abnormal. Individuals were divided into three groups: group $1-$ normal autonomic function (NAF; normal autonomic function tests and normal BRS); group 2-SAN (normal autonomic function tests but abnormal BRS) [6]; and group 3-established CAN (abnormal autonomic function and BRS tests).

\section{Adrenaline infusions}

Participants were admitted in the morning. Blood glucose was maintained between 4 and $15 \mathrm{mmol} / 1$ using a low dose intravenous infusion of insulin (Human Actrapid, Novo Nordisk Pharmaceuticals, Crawley, UK) or boluses of $20 \%$ glucose (Freeflex, Fresenius Kabi, Runcorn, UK). Arterialised venous blood samples were collected from a retrograde cannula with the hand placed in a heated chamber at $50^{\circ} \mathrm{C}$. The participants then received an incremental adrenaline (epinephrine) infusion: saline $(0.9 \% \mathrm{NaCl})$ infusion for the first hour, followed by adrenaline infusion at $0.01 \mu \mathrm{g} \mathrm{kg}^{-1} \mathrm{~min}^{-1}$ for the second hour, $0.03 \mu \mathrm{g} \mathrm{kg}^{-1} \mathrm{~min}^{-1}$ for the third hour and finally $0.06 \mu \mathrm{g} \mathrm{kg}^{-1} \mathrm{~min}^{-1}$ for $30 \mathrm{~min}$.

Plasma adrenaline, serum potassium, BP and heart rate were measured at baseline, at $30 \mathrm{~min}$ intervals during the study and $30 \mathrm{~min}$ after completion of the infusion (see ESM Methods: Biochemical analysis).

\section{ECG measurements}

Five minute high resolution ECGs were recorded at $30 \mathrm{~min}$ intervals to determine parameters of cardiac repolarisation: $\mathrm{QT}_{\mathrm{c}}, \mathrm{T}$ wave amplitude and $\mathrm{T}$ wave area symmetry ratio. ECG was recorded from three bipolar orthogonal electrodes and parameters of cardiac repolarisation were extracted from the combined composite wave. These included the $\mathrm{QT}_{\mathrm{c}}$ interval duration and parameters describing the morphology of the T wave: T wave area symmetry ratio [8] and T wave amplitude normalised to baseline values. The Hodges formula was used to correct QT for heart rate $\left(\mathrm{QT}_{\mathrm{c}}\right)$. See ESM Methods: ECG measurements for further details.

\section{Statistical analysis}

Adrenaline, potassium and cardiac repolarisation responses during the adrenaline infusion were described by summary measures for comparison between groups: AUC, time of the maximum (or minimum), absolute maximum (minimum), largest change from baseline and overall standardised variability. Groups were compared using analysis of variance where the underpinning assumptions were met or Kruskal-Wallis test. Data are presented as mean (SD) or mean $(95 \%$ CI). A $p$ value $<0.05$ was classed as significant. Statistical analysis was performed using SPSS 22 (IBM, Armonk, NY, USA). For details and power analysis see ESM Methods: Statistical analysis.

\section{Results}

\section{Participant characteristics}

Ten participants with NAF, seven with SAN and five with CAN participated in the study. Their mean age (SD) was 37 (6), 30 (6) and 38 (5) years, respectively ( $p=0.04)$, their BMI (SD) was $24(2), 26(3)$ and $29(5) \mathrm{kg} / \mathrm{m}^{2}$, respectively ( $p=$ 0.05 ) and the male to female ratio was $8 / 2,6 / 1$ and $0 / 5$, respectively $(p=0.005)$. Baseline adrenaline levels $(\mathrm{SD})$ were different across the groups: $0.38(0.17), 0.28(0.16)$ and $0.21(0.07)$ $\mathrm{nmol} / \mathrm{l}$ in the NAF, SAN and CAN groups, respectively $(p=$ 0.04) (ESM Table 1).

\section{Biochemical analysis}

Plasma adrenaline rose in all groups with no differences between the groups (Fig. 1a and ESM Table 2). Mean (95\% CI) baseline adrenaline concentration for all participants was $0.34(0.25,0.42) \mathrm{nmol} / \mathrm{l}$ and reached a maximum $4.78(4.08$, $5.47) \mathrm{nmol} / \mathrm{l}$ at $210 \mathrm{~min}$. Serum potassium fell comparably in all groups (ESM Table 3). Mean baseline potassium concentration for all participants was $4.19(4.04,4.35) \mathrm{mmol} / \mathrm{l}$ and decreased to $3.21(3.10,3.33) \mathrm{mmol} / \mathrm{l}$ at $210 \mathrm{~min}$.

\section{Physiological variables}

Baseline heart rate was $68(95 \%$ CI 60,76$)$ bpm for the NAF group, $73(59,87) \mathrm{bpm}$ for the SAN group and $84(78,91)$ bpm for the CAN group ( $p=0.06$, ESM Table 4$)$. Heart rate increase from baseline was different across the groups: $22(13$, $32) \mathrm{bpm}$ in the CAN compared with $11(7,15) \mathrm{bpm}$ in the NAF and $10(3,18) \mathrm{bpm}$ in the SAN groups $(p=0.01)$. Systolic and diastolic BP and their changes were comparable (ESM Table 1 and ESM Results: Physiological variables). 


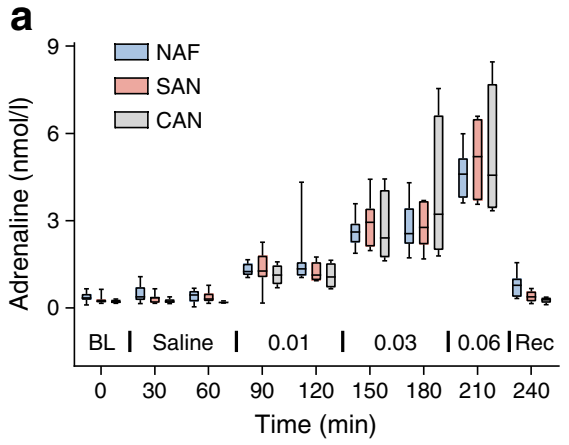

Fig. 1 Plasma adrenaline concentration (a) and $\mathrm{QT}_{\mathrm{c}}$ interval duration (b) during adrenaline infusion for participants with NAF, SAN and CAN. Data are presented using boxplots with lines at quartiles and whiskers

\section{Changes in ventricular repolarisation}

ECG waveforms at baseline and during adrenaline infusion are presented in Fig. 2 for one individual with NAF (Fig. 2a) and one with CAN (Fig. 2b). There was a decrease in T wave amplitude and the $\mathrm{T}$ waves became more symmetric with increased dose of adrenaline. In CAN the highest dose of adrenaline resulted in notched $\mathrm{T}$ waves (Fig. $2 \mathrm{~b}, \mathrm{~d}$ ) and the abnormal changes in morphology started at lower dose of adrenaline (Fig. 2d).

$\mathrm{QT}_{\mathrm{c}}$ interval duration Baseline $\mathrm{QT}_{\mathrm{c}}$ was 382 (95\% CI 374, $390) \mathrm{ms}$ in the NAF, $378(363,393) \mathrm{ms}$ in the SAN and 392 $(367,417) \mathrm{ms}$ in the CAN groups $(p=0.31)$ (Fig. $1 \mathrm{~b}$ and ESM b

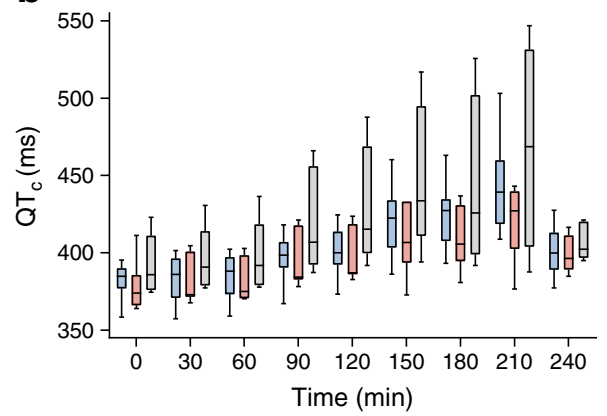

showing the full range. The adrenaline infusion protocol is displayed in an inset (a, bottom), showing adrenaline infusion rate in $\mu \mathrm{g} \mathrm{kg}^{-1} \mathrm{~min}^{-1}$. BL, baseline; Rec, recovery

Table 1). $\mathrm{QT}_{\mathrm{c}}$ in all groups lengthened comparably with adrenaline infusion (ESM Table 5). The longest $\mathrm{QT}_{\mathrm{c}}$ was 444 (422, 463) ms (NAF), $422(402,437) \mathrm{ms}(\mathrm{SAN})$ and $470(402,519)$ ms (CAN) $(p=0.09)$.

Normalised T wave amplitude and T wave area symmetry ratio Amplitude of the $T$ wave progressively decreased across all groups to about $50 \%$ of its baseline value (ESM Table 6). Time of minimum was different across the groups: 201 (95\% CI 187, 215) $\min (\mathrm{NAF}), 176(151,201) \mathrm{min}(\mathrm{SAN})$ and 120 $(29,211) \min (\mathrm{CAN})(p=0.02)$. The baseline $\mathrm{T}$ wave area symmetry ratio was comparable in the three groups (ESM Table 7). During the adrenaline infusion the T waves became more symmetric (decrease in symmetry ratio). Symmetry ratio
Fig. 2 Typical composite ECG waveforms at baseline and during adrenaline infusion at 0.01 and $0.03 \mu \mathrm{g} \mathrm{kg}^{-1} \min ^{-1}(\mathbf{a}, \mathbf{b})$. Changes in $\mathrm{T}$ wave morphology are shown for a participant with NAF (a) and established CAN (b). (c, d) ECG waveforms for the above participants are shown at all stages of the infusion protocol with different colours indicating the progressive rate of infusion. ECG T waves during adrenaline infusion demonstrate abnormal $\mathrm{T}$ wave morphology appearing to a greater extent and at lower dose of adrenaline in an individual with CAN (d) compared with NAF (c). The amplitude is normalised to baseline for each participant. The adrenaline infusion protocol is displayed below (d), showing adrenaline infusion rate in $\mu \mathrm{g}$ $\mathrm{kg}^{-1} \min ^{-1}$. BL, baseline
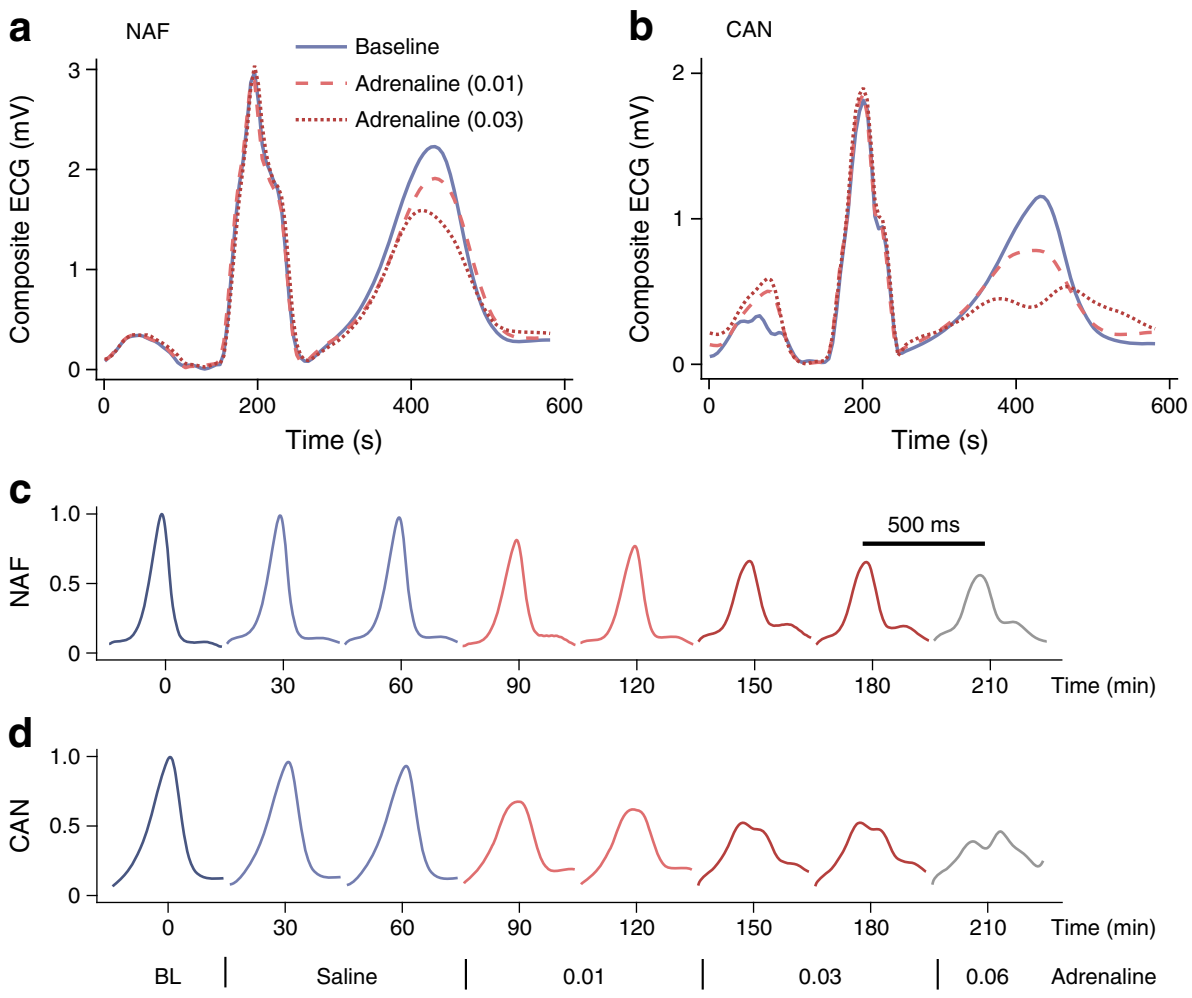
equal to number 1 indicates perfect symmetry around the peak of the T wave. AUC was lowest in the CAN group (1.19 [95\% CI 1.12, 1.25]) compared with the NAF $(1.36[1.19,1.53])$ and SAN $(1.39[1.22,1.56])$ groups $(p=0.04)$. Time of minimum was shortest in the CAN group $(p=0.01)$.

\section{Discussion}

In this study, we used the infusion of adrenaline as a standardised sympathetic stimulus to investigate the effect on cardiac repolarisation among type 1 diabetic individuals with normal autonomic function, subclinical autonomic neuropathy and established autonomic neuropathy. We noted significant changes in T wave morphology in the CAN group.

QT interval correction by adjusting for heart rate can lead to an artefactual increase in QT intervals, especially at high heart rates. Thus the morphology parameters used in this study better characterise the overall repolarisation characteristics since they are less rate dependent [8]. The maximum changes in these parameters occurred at lower infused adrenaline concentrations in individuals with CAN despite comparable adrenaline levels. We also noted abnormal notched $\mathrm{T}$ waves in the CAN group. These changes might be explained by denervation adrenergic hypersensitivity, a phenomenon that is well described in diabetic autonomic neuropathy [9]. We found no evidence that the response to adrenaline infusion was different in the SAN group compared with the NAF group. This might be due to a functional defect in SAN in contrast to a structural one in CAN.

We did not reach target sample sizes in the SAN and CAN groups despite screening over 90 potential participants. Established autonomic neuropathy is relatively uncommon in young individuals with type 1 diabetes and patients with ischaemic heart disease and renal disease were not included for safety/ethical reasons. Thus, we cannot exclude the possibility of a type 2 error. Imbalanced sex and BMI characteristics could affect some results of our study. While $\mathrm{QT}_{\mathrm{c}}$ is influenced by sex, $T$ wave symmetry is rate- and sex-independent and less likely to be affected [8]. No corrections for multiple testing could lead to inflated false positive observations in this study. The statistical data, however, were not interpreted as definite but rather indicative of repolarisation measures that best describe and classify the changes during adrenaline infusion.

In conclusion, type 1 diabetic individuals with CAN may be more vulnerable to cardiac arrhythmias when exposed to sympathoadrenal challenges. Screening for autonomic dysfunction using bedside tests could identify patients with diabetes at increased risk of cardiac arrhythmia during hypoglycaemia. Further studies with larger numbers and better balanced groups are required to confirm our findings. Those affected might theoretically benefit from $\beta$-blockers although such a treatment might also have the potential to increase the risk of hypoglycaemia in those with impaired hypoglycaemia awareness.

Acknowledgements We would like to acknowledge the patients who participated, diabetes research nurses S. Hudson and M. Cunningham and the staff of the Clinical Research Facility, Royal Hallamshire Hospital, Sheffield for their assistance with the studies. This is a summary of independent research funded by Diabetes UK and carried out at the National Institute for Health Research (NIHR) Sheffield Clinical Research Facility. The views expressed are those of the author(s) and not necessarily those of Diabetes UK, the NHS, the NIHR or the Department of Health.

Data availability The datasets generated during and/or analysed during the current study are available from the corresponding author on reasonable request.

Funding This research was supported by a grant from Diabetes UK RB113202.

Authors' relationships and activities SRH has served as a consultant or speaker for Lilly, Novo Nordisk, Takeda, Boehringer Ingelheim, Mannkind, Sanofi Aventis, Zealand Pharma and UN-EEG. The other authors declare that there are no relationships or activities that might bias, or be perceived to bias, their work.

Contribution statement $\mathrm{AB}$ analysed the data and drafted the manuscript. EC and EJR acquired the data and drafted the manuscript. JF, IAM and JLBM analysed the data. PJS designed the study. SRH conceived and designed the study. All authors contributed to critical revision of the work and all approved the final version of the manuscript. $\mathrm{SRH}$ is the guarantor of this work.

Open Access This article is licensed under a Creative Commons Attribution 4.0 International License, which permits use, sharing, adaptation, distribution and reproduction in any medium or format, as long as you give appropriate credit to the original author(s) and the source, provide a link to the Creative Commons licence, and indicate if changes were made. The images or other third party material in this article are included in the article's Creative Commons licence, unless indicated otherwise in a credit line to the material. If material is not included in the article's Creative Commons licence and your intended use is not permitted by statutory regulation or exceeds the permitted use, you will need to obtain permission directly from the copyright holder. To view a copy of this licence, visit http://creativecommons.org/licenses/by/4.0/.

\section{References}

1. O'Brien IAD, McFadden JP, Corrall RJM (1991) The influence of autonomic neuropathy on mortality in insulin-dependent diabetes. QJMed 79(290):495-502

2. Pop-Busui R (2010) Cardiac autonomic neuropathy in diabetes: a clinical perspective. Diabetes Care 33(2):434-441. https://doi.org/ $10.2337 / \mathrm{dc} 09-1294$

3. Weston PJ, Gill GV (1999) Is undetected autonomic dysfunction responsible for sudden death in type 1 diabetes mellitus? The 'dead in bed' syndrome revisited. Diabet Med 16(8):626-631. https://doi. org/10.1046/j.1464-5491.1999.00121.x 
4. Lee SP, Yeoh L, Harris ND et al (2004) Influence of autonomic neuropathy on QTc interval lengthening during hypoglycemia in type 1 diabetes. Diabetes 53(6):1535-1542. https://doi.org/10.2337/ diabetes.53.6.1535

5. O'Brien IAD, O'Hare P, Corrall RJM (1986) Heart rate variability in healthy subjects: effect of age and the derivation of normal ranges for tests of autonomic function. Br Heart J 55(4):348-354. https://doi. org/10.1136/hrt.57.1.109-a

6. Frattola A, Parati G, Gamba P et al (1997) Time and frequency domain estimates of spontaneous baroreflex sensitivity provide early detection of autonomic dysfunction in diabetes mellitus. Diabetologia 40(12):1470-1475. https://doi.org/10.1007/ s001250050851
7. Kardos A, Watterich G, de Menezes R, Csanády M, Casadei B, Rudas L (2001) Determinants of spontaneous baroreflex sensitivity in a healthy working population. Hypertension 37(3):911-916

8. Merri M, Benhorin J, Alberti M, Locati E, Moss AJ (1989) Electrocardiographic quantitation of ventricular repolarization. Circulation 80(5):1301-1308. https://doi.org/10.1161/01.CIR.80.5. 1301

9. Hilsted J, Richter E, Madsbad S et al (1987) Metabolic and cardiovascular responses to epinephrine in diabetic autonomic neuropathy. N Engl J Med 317(7):421-426. https://doi.org/10.1056/ NEJM198708133170705

Publisher's note Springer Nature remains neutral with regard to jurisdictional claims in published maps and institutional affiliations. 